\title{
Quantifying cumulative impacts of human pressures on the marine environment: a geospatial modelling framework
}

\author{
V. Stelzenmüller*, J. Lee, A. South, S. I. Rogers \\ Centre for Environment, Fisheries and Aquaculture Science (Cefas), Pakefield Road, Lowestoft NR33 OHT, UK
}

\begin{abstract}
Worldwide increasing pressure on the marine environment requires integrated and ecosystem-based management, and a sound understanding of cumulative impacts of human pressure. As yet, the quantification of risk of cumulative impact remains a difficult task in practice. We developed a geospatial modelling framework to group data on the spatial distribution and intensity of human activities by generic pressure. The impact of those pressures was mapped by accounting for the sensitivity of UK marine landscapes to those pressures. With the help of GIS-based multi-criteria analysis, we developed 4 different scenarios to quantify risk of cumulative impacts that accounted for different importance of ranked pressures (equal, linear and logistic decrease), including a simulated expert consultation. Finally, we assessed the sensitivity of the scenario outcomes to changes to input parameters and compared model outcomes. The risk assessment framework exposed both a wide range of possible modelled scenarios and uncertainties, but all scenarios revealed similar locations with an increased risk of cumulative impacts. Results showed that the logistic weighting scheme was very sensitive to changes in the importance and ranking of pressures in comparison to the linear weighting scheme. For marine planning the use of a weighting scheme with more constrained values should be used in conjunction with a sensitivity analysis to determine the order of input parameters. Once a more comprehensive geodatabase becomes available our standardised framework can be applied to support both the development of sustainable marine plans in practise and the prioritisation of different uses.
\end{abstract}

KEY WORDS: GIS-MCA · GIS-based multi-criteria analysis - Fuzzy sets - Human pressures · Sensitivity $\cdot$ Impact $\cdot$ Marine landscapes $\cdot$ Marine planning

\section{INTRODUCTION}

Worldwide increasing pressure on the marine environment, due to development of new technologies such as offshore renewable energy, and the conflicting interests that occasionally result, requires integrated and ecosystem-based management. An ecosystem approach to management promotes the integrated management of human uses while maintaining ecosystem goods and services (Leslie \& McLeod 2007, Ruckelshaus et al. 2008), but must address interactions between a range of human activities which vary in their intensity, footprint (Eastwood et al. 2007) and impact on the marine environment (Crain et al. 2008). One tool that supports such an ecosystem approach to management is marine spatial planning (Douvere 2008). Thus, an understanding of the risk of cumulative impacts of numerous activities is crucial for the development of a marine spatial plan.

In general, determining the impact of a human activity on an ecosystem component requires both a method for translating human activities into ecosystem-specific pressures, and a measure of sensitivity of ecosystem components to those pressures. Human activities have already been grouped by the type of pressure, such as contamination, abrasion, extraction, that they exert on 
ecosystem components (Eastwood et al. 2007). Following Zacharias \& Gregr (2005), we define the impact of a pressure on an ecosystem component as a function of the spatial footprint and intensity of a pressure, and a measure of the degree to which an ecosystem component responds to a pressure (sensitivity).

The risk of an impact at a certain spatial location is therefore a function of a measure of intensity of human activities causing the same generic pressure and the sensitivity of the ecosystem component to this generic pressure. However, an ecosystem approach to marine management that makes use of marine spatial planning needs to consider cumulative and interactive impacts of several human activities (Evans \& Klinger 2008, Halpern et al. 2008a). Cumulative impacts can be described as the combined impact of multiple pressures over space and time (MacDonald 2000).

The risk and consequences of cumulative impacts on ecosystems due to the presence of multiple pressures are discussed in a number of theoretical and practical ecological studies (Folt et al. 1999, Folke et al. 2004, Vinebrooke et al. 2004, Christensen et al. 2006, Halpern et al. 2008b). The quantification of cumulative impacts still remains a difficult task for applied ecology and conservation science (see Darling \& Côté 2008 and references therein). If multiple pressures act independently within a system, cumulative impacts may be modelled as the additive accumulation of impacts of individual pressures (Halpern et al. 2007, 2008a, Ban \& Alder 2008). However, a common ecological paradigm is that multiple pressures can act in synergy (Folt et al. 1999, Folke et al. 2004, Christensen et al. 2006, Crain et al. 2008). Synergistic effects occur when the combined impact of several pressures is greater than the algebraic sum of the impacts of the individual pressures. In contrast, antagonistic effects occur when the combined impact of multiple pressures is less than the sum of the individual impacts of the pressures (Folt et al. 1999). Recent studies exploring the nature of interaction of multiple pressures show that cumulative impacts are additive (i.e. are summed) for pairs of pressures (Crain et al. 2008) and that synergistic effects are generally more common than additive ones (Darling \& Côté 2008). This highlights the need to evaluate the complexity and range of uncertainty in assessing cumulative impacts of human pressures, especially for the development of sustainable marine spatial plans.

Recent advances in the quantification of risks of cumulative impacts in marine systems have been demonstrated by Halpern et al. (2007, 2008b). They combined the spatial information on the occurrences and intensity of human activities with a measure of sensitivity of various ecosystem components to the activities and assumed additive cumulative impacts.
Inspired by their work, we developed a geospatial modelling framework which assesses a range of models that quantify the risk of cumulative impacts on UK marine habitats by altering the importance of single pressures that lead to numerical results other than addition of single pressures. Moreover, our framework enables the evaluation of both the uncertainty at different stages of the assessment and the sensitivity to respective model parameters.

Multi-criteria analysis (MCA) is a decision-making tool that allows for comparative assessment of alternative scenarios. It generates a set of alternative outcomes based on multiple criteria, where the outputs are quantified indicators of the extent to which different objectives are realised (Malczewski 1999). The set of alternative outcomes can then be evaluated, but the final decision will be taken by the decision maker. The integration of MCA in Geographical Information System (GIS), i.e. a spatially explicit MCA, is a major contribution to the development of tools for integrated management (Jiang \& Eastman 2000, Malczewski 2006). In the marine environment, GIS-MCA has been employed to aid zoning within marine protected areas (Villa et al. 2002), across national borders (Portman 2007) and in coastal areas (Kitsiou et al. 2002). It has also been applied to identify candidate sites for protection based on multiple objectives (Wood \& Dragicevic 2007).

First, our algorithm combined standardised data describing locations and/or intensities of human activities (Eastwood et al. 2007, Stelzenmüller et al. 2008) with a measure of the sensitivity of the UK marine landscapes (Connor et al. 2006) to the pressures of these activities. We then applied a weighting to these impact layers and combined them to model 4 scenarios quantifying the risk of cumulative impacts on the UK continental shelf. The computed cumulative impact scenarios assumed (1) equal importance of all pressures, (2) linear decrease of importance of subsequent pressures, (3) logistic decrease of importance of pressures, and (4) ranking of importance of pressures by simulating an expert consultation. We were also able to assess the sensitivity of the outcomes of the analysis to changes in the input parameters. If a small change in the rank order of an input parameter leads to a large change in the results then the outcome is deemed to be sensitive to that parameter and may indicate a need for more accurate determination of the parameter, or of consideration of the alternative scenarios in the decision making process. We compared and interpreted the different models based on the definition of additive effects, and the final outcome in terms of potential synergistic and antagonistic effects of multiple impacts. The results and the use of our framework in marine planning is discussed. 


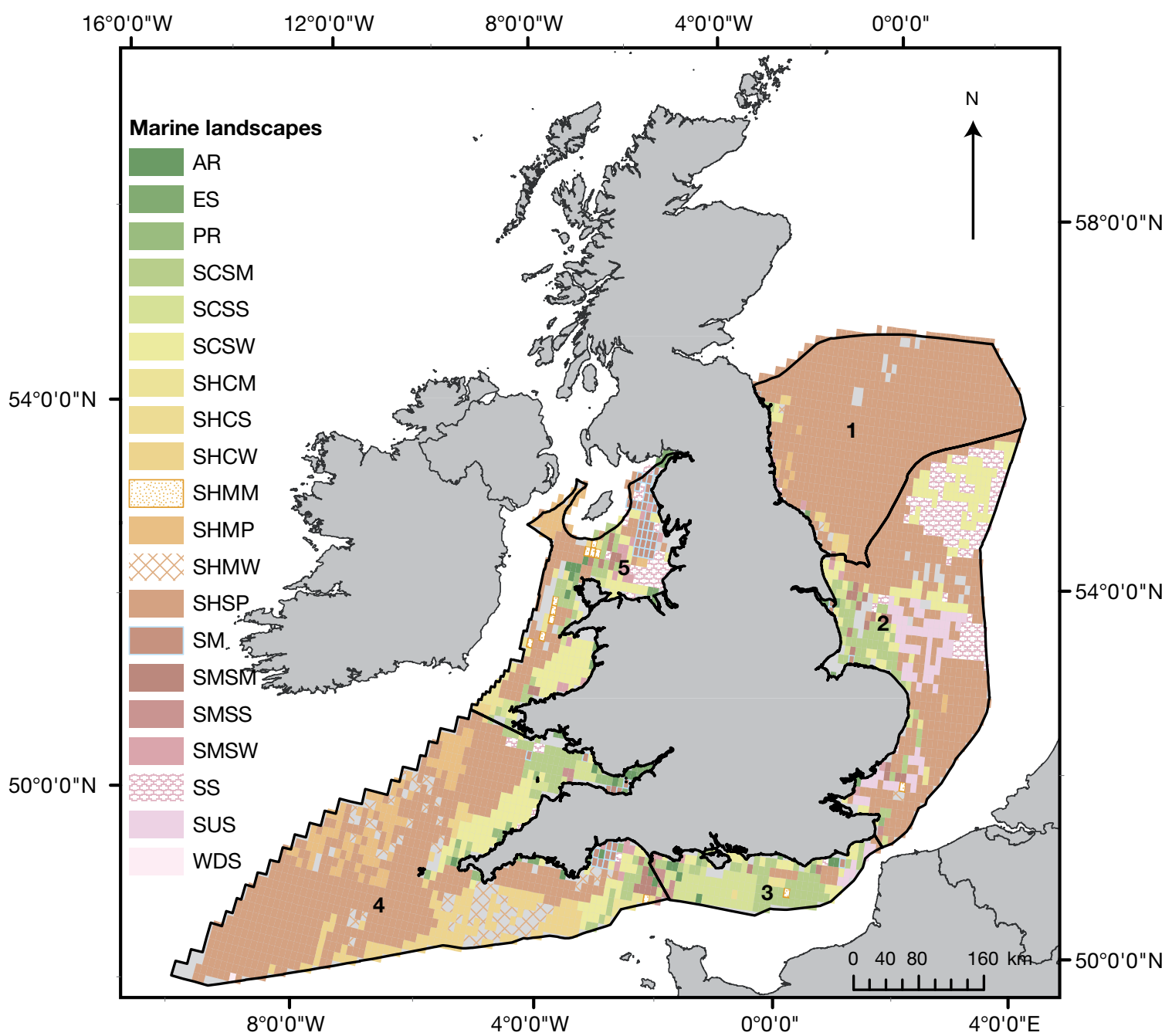

Fig. 1. Study area comprising the waters of the United Kingdom (England and Wales), the northern North Sea, southern North Sea, eastern English Channel, western English Channel, Celtic Sea, and southwest approaches, and the Irish Sea and North Channel limited by the borders of the reporting regions 1 to 5. The codes for the marine landscape categories, modified from Connor et al. (2006), correspond with the categories listed in Table 2

\section{METHODS}

Study area and geospatial modelling framework. Driven by the Marine Bill, the UK is currently developing an approach to marine planning that aims to support the achievement of high level objectives such as good environmental status (EU 2008), and to integrate current activities as well as future uses. As planning boundaries are not yet defined, our study area covered the waters of the United Kingdom (England and Wales) (Fig. 1), limited by the boundaries of the reporting areas (RAs) 1 to 5 (Defra 2005) used by the UK government for environmental status reporting. Within English and Welsh waters, Eastwood et al. (2007) identified demersal fishing as the most important human activity with respect to the spatial footprint and relative impact on the seabed. Next in importance was the installation of oil and gas infrastructure, such as wellheads, platforms, cables and pipelines, followed by aggregate extraction and wrecks.

The first step of our geospatial modelling framework comprised the mapping of the footprint and intensity of selected human activities for the study area in a GIS (ESRI ArcGIS v9.2), the grouping of activities by their generic pressures and the subsequent computation of pressure maps (Fig. 2). In the next step, these generic pressure maps were transformed and standardised using fuzzy logic. We considered the UK marine landscapes as representative ecosystem components to test the model. For each landscape, a measure of sensitivity 
Fig. 2. Conceptual geospatial modelling framework to assess 4 different scenarios that model the risk of cumulative impact (CI) of human pressures

to the respective pressure was produced. Thus, the combination of the measures of pressure and sensitivity enabled the computation of the respective impact layers. Afterwards, a GIS-MCA was used to construct 4 different scenarios to quantify the risk of cumulative impacts of human pressures. The final step of the framework was the evaluation of the robustness and sensitivity of the different scenario outcomes. In the following, we describe each step in more detail.

Human pressures and fuzzy sets. We selected a suite of human activities for which geospatial data with a spatial and temporal coverage for the UK continental shelf were available (in the geographic coordinate system WGS84). Following the approach of Eastwood et al. (2007) and Stelzenmüller et al. (2008), we mapped in a GIS the occurrence of human activities, considering where possible both their spatial footprint and intensity (see Table 1 for details). Following a recent study by Eastwood et al. (2007), we grouped human activities by their generic pressure categories, which are also listed in the Marine Strategy Framework Directive (MSFD; EU 2008) (see Table 1). In the GIS, we merged activities exerting the same pressure on the marine environment into a single pressure layer. We superimposed a vector grid with a cell size of 0.05 by 0.1 degrees, which was a compromise between the spatial resolution of the data used and computation time at the scale of our study area. Then we reprojected geodata to an equal area reference system to produce in a following step a series of 6 pressure layers (abrasion, extraction, localised hydrocarbon contamination, obstruction, siltation and smothering) based on the proportion of a grid cell affected by the footprint and/or intensity of all the human activities exerting the same pressure (for abrasion and extraction, the average pressure per grid cell was calculated for the respective period of time considered; see detailed description in Table 1).

Although all pressure maps were computed as a proportion of a grid cell impacted by a given pressure, different units, measures and levels of generalisation were evident in the data used to describe each human activity. As a result, they all possess a level of uncertainty. Thus, a standardisation of multiple raster layers (criterion layers) is required to maintain the validity of GIS-MCA outputs. Deterministic criteria are most frequently standardised by means of linear scale transformations, while issues relating to the uncertainty and imprecision of the underlying criterion layers can be addressed by fuzzy set theory and fuzzy logic (Burrough \& McDonnell 1998, Fisher 2000, Malczewski 2004). The central concept of fuzzy set theory is the membership function $(M F)$, which numerically represents the degree to which a given measure of criteria $(z)$ within a grid cell belongs to a fuzzy set. In other words, this membership function standardises each raster cell of the layer to a measure of the possibility of belonging to the entire set along a continuous scale of 0 to 1 (see detailed description in Burrough \& McDonnell 1998). To standardise each grid cell, we used an 
Table 1. Human activities included in the risk assessment framework with respective descriptions of how the proportion of a grid cell ( 0.05 by 0.1 decimal degrees) impacted by the activities has been calculated in the GIS. Generic human pressure categories associated with each activity are listed (ABR: abrasion; OBS: obstruction; SMO: smothering; HCC: hydrocarbon contamination;

EXT: extraction; SIL: siltation [sediment plume]). VMS: Vessel Monitoring System; EMS: electronic monitoring system

\begin{tabular}{|c|c|c|}
\hline Human activity & Geodata on human activities & $\begin{array}{l}\text { Pressure } \\
\text { category }\end{array}$ \\
\hline $\begin{array}{l}\text { Fishing activity with } \\
\text { a physical impact on } \\
\text { the seabed }\end{array}$ & $\begin{array}{l}\text { The average fishing pressure as proportion of a grid cell fished (2001 to 2006) } \\
\text { derived from standardised VMS data was summarised for each grid cell } \\
\text { (Eastwood et al. 2007, Stelzenmüller et al. 2008). }\end{array}$ & $\mathrm{ABR}$ \\
\hline $\begin{array}{l}\text { Renewable energy } \\
\text { (licensed areas for } \\
\text { wind farm) }\end{array}$ & $\begin{array}{l}\text { The area of polygons of currently (2008) licensed wind farm areas as proportion } \\
\text { of a gird cell impacted. }\end{array}$ & $\begin{array}{l}\text { OBS, } \\
\text { SMO }\end{array}$ \\
\hline $\begin{array}{l}\text { Oil and gas } \\
\text { development (well- } \\
\text { heads and platforms) }\end{array}$ & $\begin{array}{l}\text { Geodata on current (2008) oil and gas infrastructure were used and a buffer } \\
\text { distance of } 15 \mathrm{~m} \text { was applied to oil and gas platforms and a buffer of } 50 \mathrm{~m} \text { to } \\
\text { wellheads (see Eastwood et al. 2007). Thus the proportion of a grid cell impacted } \\
\text { by the activity was calculated. }\end{array}$ & $\begin{array}{l}\text { OBS, } \\
\text { SMO, } \\
\text { HCC }\end{array}$ \\
\hline Cables & $\begin{array}{l}\text { Geodata on current (2008) cables were used and a buffer distance of } 0.09 \mathrm{~m} \\
\text { (Eastwood et al. 2007) was applied to calculate the proportion of a grid } \\
\text { cell impacted. }\end{array}$ & OBS \\
\hline Pipelines & $\begin{array}{l}\text { Geodata on current (2008) pipelines were used and a buffer distance of } 0.7 \mathrm{~m} \text { was } \\
\text { applied to pipelines as their diameter can vary between } 0.76 \text { and } 1.37 \mathrm{~m} \text { (Gerwick } \\
\& \text { Gerwick 2007). The proportion of a grid cell impacted was then calculated. }\end{array}$ & $\begin{array}{l}\text { OBS, } \\
\text { HCC }\end{array}$ \\
\hline $\begin{array}{l}\text { Aggregate extraction } \\
\text { (licensed activity and } \\
\text { modelled area of } \\
\text { influence) }\end{array}$ & $\begin{array}{l}\text { For each grid cell the average dredging activity (2001 to } 2007 \text { ) as h per } 50 \times 50 \mathrm{~m} \\
\text { dredged, derived from EMS data (Foden et al. } 2009 \text { ) was summarised. The } \\
\text { sediment plume based on the dredging activity of } 2004 \text { was modelled (Vaz et al. } \\
2008 \text { ) and the proportion of a grid cell impacted was calculated. }\end{array}$ & $\begin{array}{l}\text { EXT, } \\
\text { SIL }\end{array}$ \\
\hline Dumping grounds & $\begin{array}{l}\text { Geodata on current (2008) dumping grounds were used and the proportion of grid } \\
\text { cells impacted was calculated using the polygons of licensed dumping grounds. }\end{array}$ & SMO \\
\hline $\begin{array}{l}\text { Other constraining } \\
\text { activities (wrecks) }\end{array}$ & $\begin{array}{l}\text { Positions of wrecks (2008) were buffered by a distance of } 35.1 \mathrm{~m} \text { (Eastwood et al. } \\
2007) \text { and the proportion of a grid cell impacted was calculated. }\end{array}$ & OBS \\
\hline
\end{tabular}

asymmetric sigmoid membership function (Malczewski 2004, Wood \& Dragicevic 2007, Maina et al. 2008) modified after Burrough \& McDonnell (1998):

$$
\begin{aligned}
& M F(z)=\frac{1}{1+\left(\frac{z-a-b}{a}\right)^{2}} \text { if } 0<z<a+b \\
& M F(z)=1 \text { if } z \geq a+b
\end{aligned}
$$

with $a$ as the standard deviation and $b$ as the 0.9 quartile of the respective log-transformed measure of a pressure.

Measures of sensitivity and impact. We adopted the marine landscape categories developed by Connor et al. (2006) for our study grid and distinguished between 20 landscape categories at the scale of the grid size used (Fig. 1). Each raster cell was assigned to one marine landscape category. From an extensive literature review (Defra 2007), we extracted information on sensitivity of a marine landscape to each human activity presented on an ordinate scale (no, low, medium and high). Then we converted this measure of sensitivity from an ordinate scale to a numeric measure for sensitivity (sensitivity score) 0 (no), 0.2 (low), 0.6 (medium) and 1 (high) (Table 2). In cases where more than one human activity contributed to a pressure category, the average sensitivity across all relevant activities was calculated. This enabled us to compute for each raster cell the impact $(I)$ of a given pressure:

$$
I_{i}=P_{i} S S_{i j}
$$

with $P_{i}$ as the fuzzy standardised (0 to 1) measure of a pressure $(i=1,2, \ldots 6)$ and $S S$ as the sensitivity score (Defra 2007) of the landscape category $j(j=1,2, \ldots 20)$ for the given pressure $P_{i}$. In total, we computed 6 impact layers for the UK and Welsh waters accounting for the 6 pressure categories.

Quantification of cumulative impacts and scenario evaluation. We modelled 4 different scenarios to quantify the risk of cumulative impacts for the UK continental shelf. After constructing the impact layers, a weight was assigned to each layer reflecting its relative importance (from the user's perspective). The layers are then combined according to different decision rules (Boroushaki \& Malczewski 2008). The weighted linear combination is the decision rule most commonly used for a GIS-MCA and therefore, for all scenarios, we quantified the relative risk of cumulative impacts for each raster cell $\left(\right.$ RiskCI$\left._{\text {cell }}\right)$ as (after Malczewski 1999): 
Table 2. Sensitivity scores (SS) for the generic pressures (see Table 1) derived from an extensive literature review of impact of human activities on UK marine landscapes (Defra 2007). We converted the measure of sensitivity from an ordinate scale to a numeric scale (no $=0$, low $=0.2$, medium $=0.6$, high $=1.0$ ) and merged the human activities by their general environmental impact and averaged the impact scores per landscape (see details in 'Methods')

\begin{tabular}{|c|c|c|c|c|c|c|c|}
\hline Marine landscape & Code & $\mathrm{SS}_{\mathrm{SMO}}$ & $\mathrm{SS}_{\mathrm{OBS}}$ & $\mathrm{SS}_{\mathrm{EXT}}$ & $\mathrm{SS}_{\mathrm{SIL}}$ & $\mathrm{SS}_{\mathrm{ABR}}$ & $\mathrm{SS}_{\mathrm{HCC}}$ \\
\hline Aphotic rock & $\mathrm{AR}$ & 0.60 & 0.44 & 0.00 & 0.20 & 0.60 & 0.20 \\
\hline Estuary & ES & 0.87 & 1.00 & 1.00 & 1.00 & 0.60 & 0.20 \\
\hline Photic rock & PR & 0.60 & 0.44 & 0.00 & 0.20 & 0.60 & 0.20 \\
\hline Shallow coarse sediment plain - weak tide stress & SCSW & 0.60 & 0.52 & 1.00 & 0.60 & 0.86 & 0.20 \\
\hline Shallow mixed sediment plain - weak tide stress & SMSW & 0.60 & 0.52 & 1.00 & 0.60 & 0.86 & 0.20 \\
\hline Shallow coarse sediment plain - moderate tide stress & SCSM & 0.60 & 0.52 & 1.00 & 0.20 & 0.60 & 0.20 \\
\hline Shallow mixed sediment plain - moderate tide stress & SMSM & 0.60 & 0.52 & 1.00 & 0.20 & 0.60 & 0.20 \\
\hline Shallow coarse sediment plain - strong tide stress & SCSS & 0.47 & 0.44 & 1.00 & 0.20 & 0.33 & 0.20 \\
\hline Shallow mixed sediment plain - strong tide stress & SMSS & 0.47 & 0.44 & 1.00 & 0.20 & 0.33 & 0.20 \\
\hline Shallow mud plain & SM & 0.53 & 0.52 & 0.00 & 0.00 & 0.60 & 0.20 \\
\hline Shallow sand plain & SS & 0.53 & 0.52 & 0.60 & 0.20 & 0.46 & 0.20 \\
\hline Shelf coarse sediment plain - weak tide stress & SHCW & 0.33 & 0.36 & 1.00 & 0.60 & 0.20 & 0.20 \\
\hline Shelf mixed sediment plain - weak tide stress & SHMW & 0.33 & 0.36 & 1.00 & 0.60 & 0.20 & 0.20 \\
\hline Shelf coarse sediment plain - moderate tide stress & SHCM & 0.33 & 0.36 & 1.00 & 0.20 & 0.20 & 0.20 \\
\hline Shelf mixed sediment plain - moderate tide stress & SHMM & 0.33 & 0.36 & 1.00 & 0.20 & 0.20 & 0.20 \\
\hline Shelf coarse sediment plain - strong tide stress & SHCS & 0.33 & 0.36 & 1.00 & 0.20 & 0.20 & 0.20 \\
\hline Shelf mud plain & SHMP & 0.33 & 0.36 & 0.00 & 0.00 & 0.20 & 0.20 \\
\hline Shelf sand plain & SHSP & 0.33 & 0.36 & 0.60 & 0.20 & 0.20 & 0.20 \\
\hline Subtidal sediment bank & SUS & 0.27 & 0.36 & 0.60 & 0.20 & 0.20 & 0.20 \\
\hline Warm deep-water sand plain & WDS & 0.20 & 0.32 & 0.00 & 0.00 & 0.00 & 0.20 \\
\hline
\end{tabular}

$$
\operatorname{RiskCI}_{\text {cell }}=\sum_{j} I_{i} w_{j} \quad \text { and } \quad \sum_{j} w_{j}=1
$$

where $I_{i}$ are the impact layers and $w_{j}$ are the criterion weights, which are listed in Table 3.

In our framework, we constructed 4 scenarios (see Table 3): (1) equal importance of pressures (Scenario 1), (2) linear decrease of importance of pressures (Scenario 2), (3) logistic decrease of importance of pressures (Scenario 3) and (4) ranking of importance of pressures derived from a simulated expert consultation (Scenario 4). For Scenarios 2 and 3, we ranked pressures by their spatial extent (percentage of grid cells with a positive value for the pressure), assuming the

Table 3. Relative weights of the criterion layers (impact layers) reflecting the importance of the respective pressure categories (see Table 1) for 4 different scenarios. Scenario $1=$ equal weight, Scenario $2=$ linear weight, Scenario $3=$ logistic weights, Scenario $4 \mathrm{a}=$ simulated pairwise comparison with 25 iterations, Scenario $4 \mathrm{~b}=$ simulated pairwise comparison with 500 iterations

Pressure Scenario 1 Scenario 2 Scenario 3 Scenario 4a Scenario 4b category

\begin{tabular}{lccccc}
\hline OBS & 0.167 & 0.261 & 0.632 & 0.174 & 0.166 \\
ABR & 0.167 & 0.223 & 0.235 & 0.157 & 0.172 \\
HCC & 0.167 & 0.185 & 0.087 & 0.163 & 0.165 \\
SMO & 0.167 & 0.147 & 0.032 & 0.155 & 0.166 \\
SIL & 0.167 & 0.109 & 0.012 & 0.175 & 0.166 \\
EXT & 0.167 & 0.071 & 0.004 & 0.177 & 0.166 \\
Sum & 1 & 1 & 1 & 1 & 1 \\
& & & & & \\
\hline
\end{tabular}

greater the spatial extent of a pressure the lower the likelihood of a general recovery from the respective pressure. Thus, obstruction was ranked for Scenarios 2 and 3 as the most severe pressure and aggregate extraction as the least (see Table 3).

For the fourth scenario, we simulated a random pairwise comparison of the importance of pressures. A pairwise comparison is the basic measurement method in an analytical hierarchy process (AHP) (Saaty 1980). AHP uses weighted overlays and is of particular importance for spatial decision problems with a large number of alternatives (Saaty 1980, Boroushaki \& Malczewski 2008). Thus, the comparison of the relative importance of pairs of pressures by stakeholder consultation would generate a large number of alternatives. We ran 2 random simulations of stakeholder consultations with 25 (Scenario 4a) and 500 (Scenario 4b) iterations of pairwise comparisons of pressures using a reciprocal matrix and a scale of values from 1 to 9 reflecting the relative preferences for 2 pressure categories (e.g. 1 = equal importance, $3=$ moderate importance, $5=$ strong importance, $7=$ very strong importance, $9=$ extreme importance, 2, 4, 6, $8=$ intermediate values between adjacent scale values) (see Boroushaki \& Malczewski 2008). The relative weights for the impact layers in Scenario 4 (see Table 3) were derived by nor- 
malising the eigenvector associated with the maximum eigenvalue of the respective pairwise comparison matrix. This provides a weighting derived from the priority vector of the matrix and reflects the relative importance of each criteria as compared to all other criteria (see a detailed description in e.g. Boroushaki \& Malczewski 2008).

Scenario evaluation and comparison. We assessed the sensitivity of scenario results to variations in model input parameters and compared scenario outcomes with the help of explorative tools and GIS. First, to test the similarity of scenario outcomes, we plotted the raster values for each scenario against each other and calculated Pearson correlation coefficients. Then we conducted a sensitivity assessment of model input parameters by testing the influence of both single pressures and the relative ranking of the pressure categories on the scenario results. Hence, if a scenario result is sensitive to a change to the order of the pressures or the assigned weight, this indicates the need for a more accurate determination of the model parameters. Thus, we recalculated the scenarios by leaving out the most (obstruction; OBS) and least important pressure category (extraction; EXT). Subsequently, we used 6 sets of randomly assigned pressure categories in the weighting schemes of Scenarios 2 and 3 to assess different combinations of pressure category $\times$ weight on the quantification of cumulative impacts (Table 4). With the help of cubic smoothing splines (Hastie \& Tibshirani 1990), we explored differences between the predictions made by the 6 random sets of pressure category $\times$ weight combinations and the prediction based on ranked pressures, respectively.

Although the concept of a weighted overlay is applied to all scenarios, the value for cumulative impact for any single raster cell will differ due to the various weighting schemes applied to the individual impact layers. We used the definition of cumulative impact as a result of additive (sum of individual impacts), synergistic (greater than the algebraic sum of individual impacts) and antagonistic (lower than the algebraic

Table 4. As an example, the 6 random allocations of the pressure categories (see Table 1) to weights of Scenario (sc) 2 are shown. The same random sets were assigned to the weights of Scenario 3 (see Fig. 7)

\begin{tabular}{lcccccc}
$\begin{array}{l}\text { Scenario } 2 \\
\text { weights }\end{array}$ & sc2 r1 & sc2 r2 & sc2 r3 & sc2 r4 & sc2 r5 & sc2 r6 \\
\hline 0.261 & & & & & & \\
0.223 & OBS & HCC & EXT & EXT & SIL & SMO \\
0.185 & ABR & OBS & ABR & ABR & ABR & OBS \\
0.147 & SMO & EXT & SIL & HCC & OBS & SIL \\
0.109 & EXT & SIL & HCC & SIL & HCC & HCC \\
0.071 & HCC & ABR & OBS & SMO & SMO & EXT \\
& SIL & SMO & SMO & OBS & EXT & ABR \\
\hline
\end{tabular}

sum of individual impacts) interactions of multiple impacts to compare the model outputs (see Folt et al. 1999). We treated our Scenario 1 (equal weighting of the pressures) as the reference scenario, which can be interpreted as the quantification of cumulative impacts using the sum of equally important individual impact layers (additive interaction). Thus, if for a given raster cell, a scenario $(2,3,4 \mathrm{a}$ and $4 \mathrm{~b})$ predicted a value greater than the value of Scenario 1, the result for that raster cell is interpreted as an assumed synergistic effect. In contrast, if for a given raster cell a scenario predicted a value lower than the value of Scenario 1 the result for the raster cell is interpreted as an assumed antagonistic effect. In a GIS, we produced maps comparing the reference Scenario 1 to all other scenarios (Scenario 1 to Scenarios 2, 3, 4a and 4b).

\section{RESULTS}

\section{Scenarios of risk of cumulative impact}

The impact layer prepared using the abrasion data (ABR) (reflecting fishing activity with a physical impact on the seabed) and the habitat sensitivity scores are shown in Fig. 3. An increased impact of abrasion can be found at 2 distinct areas off the south west coast of the UK. Off the northeast coast there was a medium level of impact due to the presence of more sensitive marine landscapes (Fig. 3).

The different scenarios of cumulative impact (Fig. 4) showed generally consistent spatial patterns with an increased risk of cumulative impacts in the south east, north east and east of the study area. Scenario 3, using a logistic decrease in the importance of pressures, showed the greatest spatial deviation where the areas of increased risk off the east coast of the UK clearly differed in shape and extent from the areas predicted by Scenarios 1, 2, 4a and $4 \mathrm{~b}$.

\section{Scenario evaluation}

The pairwise correlations of the scenario raster values (Fig. 5) showed lower values of the Pearson correlation coefficient for Scenario 3 versus Scenario $4 \mathrm{a}$ (0.70) and Scenario 3 versus Scenario 1 (0.72). This underpins our visual observation of a clear spatial deviation of the Scenario 3 outcome from the other scenario results. As an example, the results of the sensitivity assessment are presented for Scenarios 2 and 3 in Fig. 6. In general, we found a good agreement between the results of Scenario 2 (Fig. 4) and the recomputed Scenario 2 results where the most and least severe pressures have been left out (Fig 6). We 


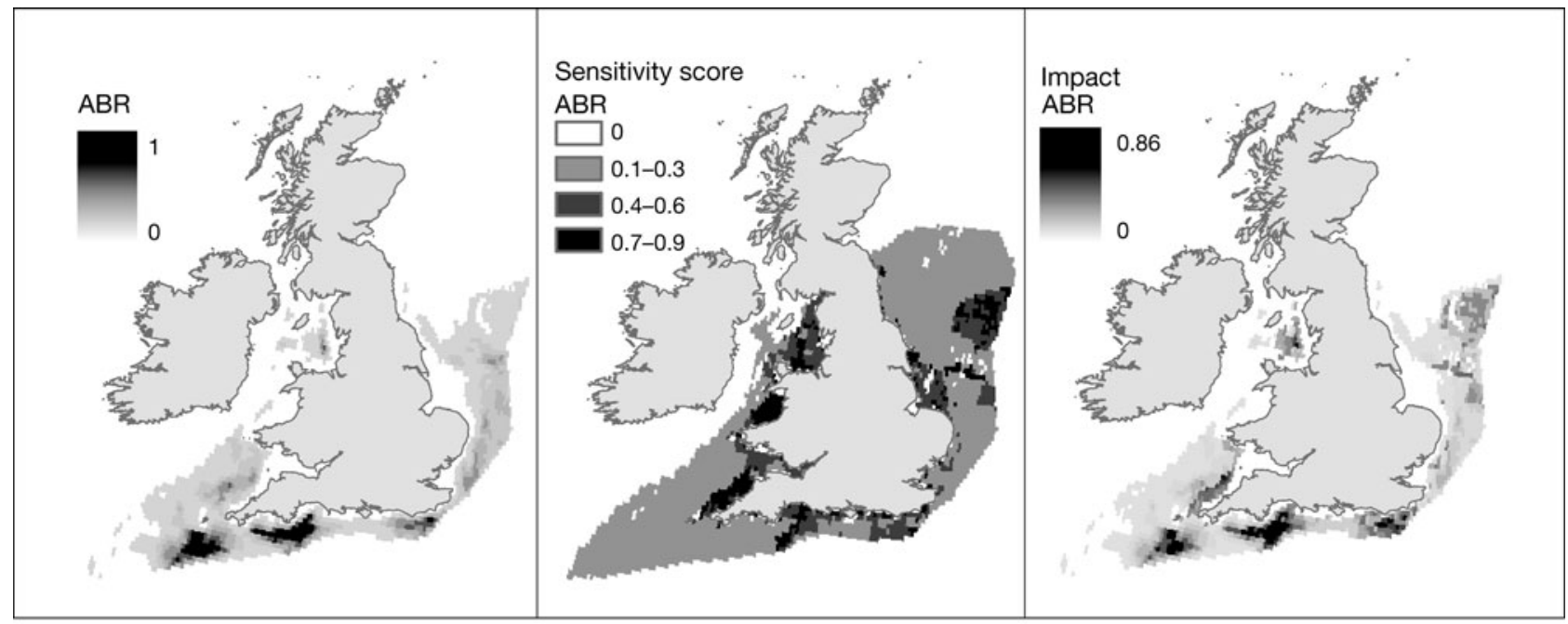

Fig. 3. Left: Abrasion (ABR) pressure map based on processed Vessel Monitoring System (VMS) data reflecting the average (2001 to 2006) fishing pressure of beam trawls, otter trawls and scallop dredgers (see Stelzenmüller et al. 2008); mid: marine landscape categories with associated measure of sensitivity (derived from Defra 2007) to abrasion; right: estimated impact of abrasion

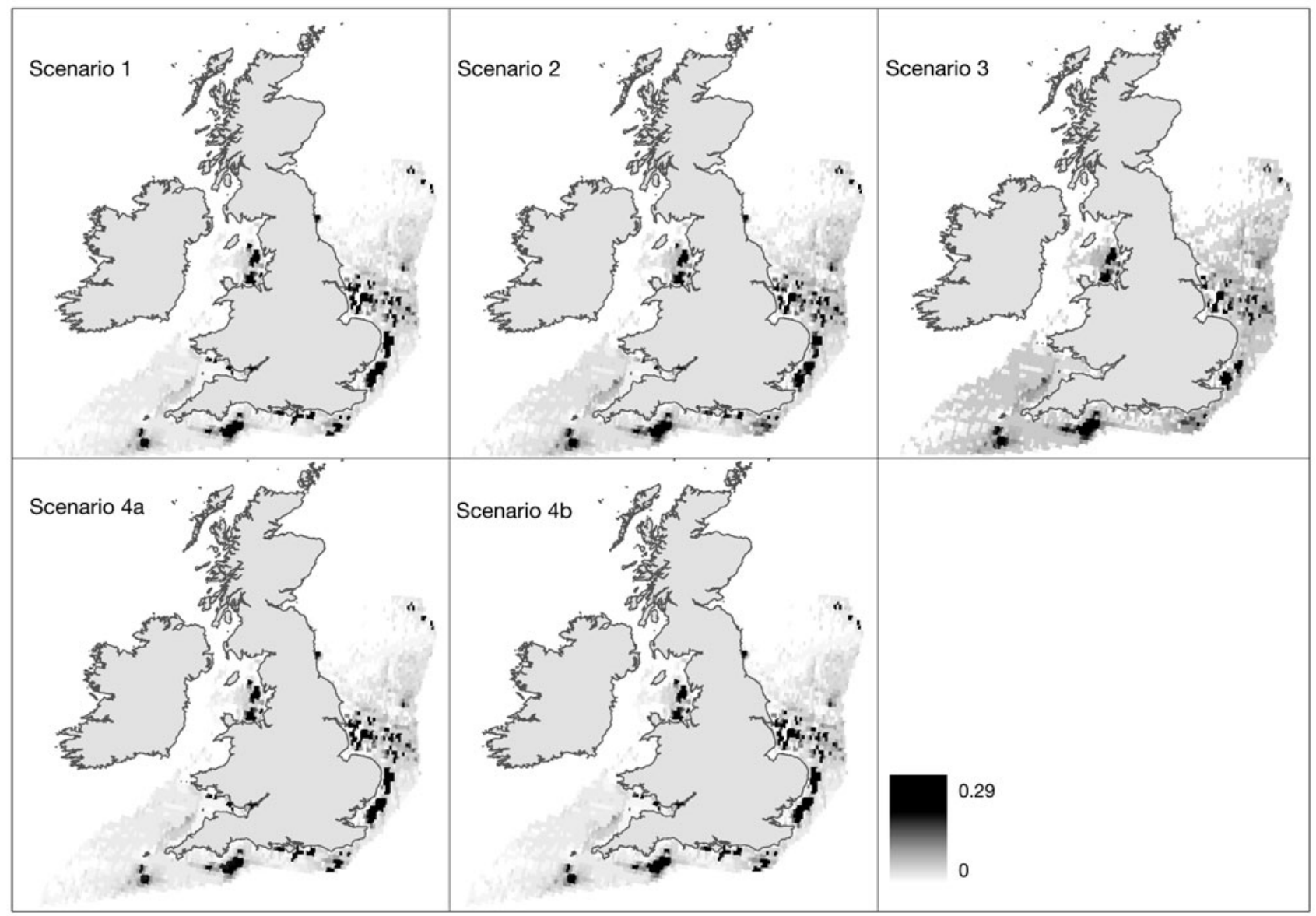

Fig. 4. Results of the 4 scenarios quantifying the risk of cumulative impacts based on the weighted overlay of the criterion layers (impact layers). The results are scaled to a qualitative scale of risk of cumulative impact (0 to 0.29 ) 


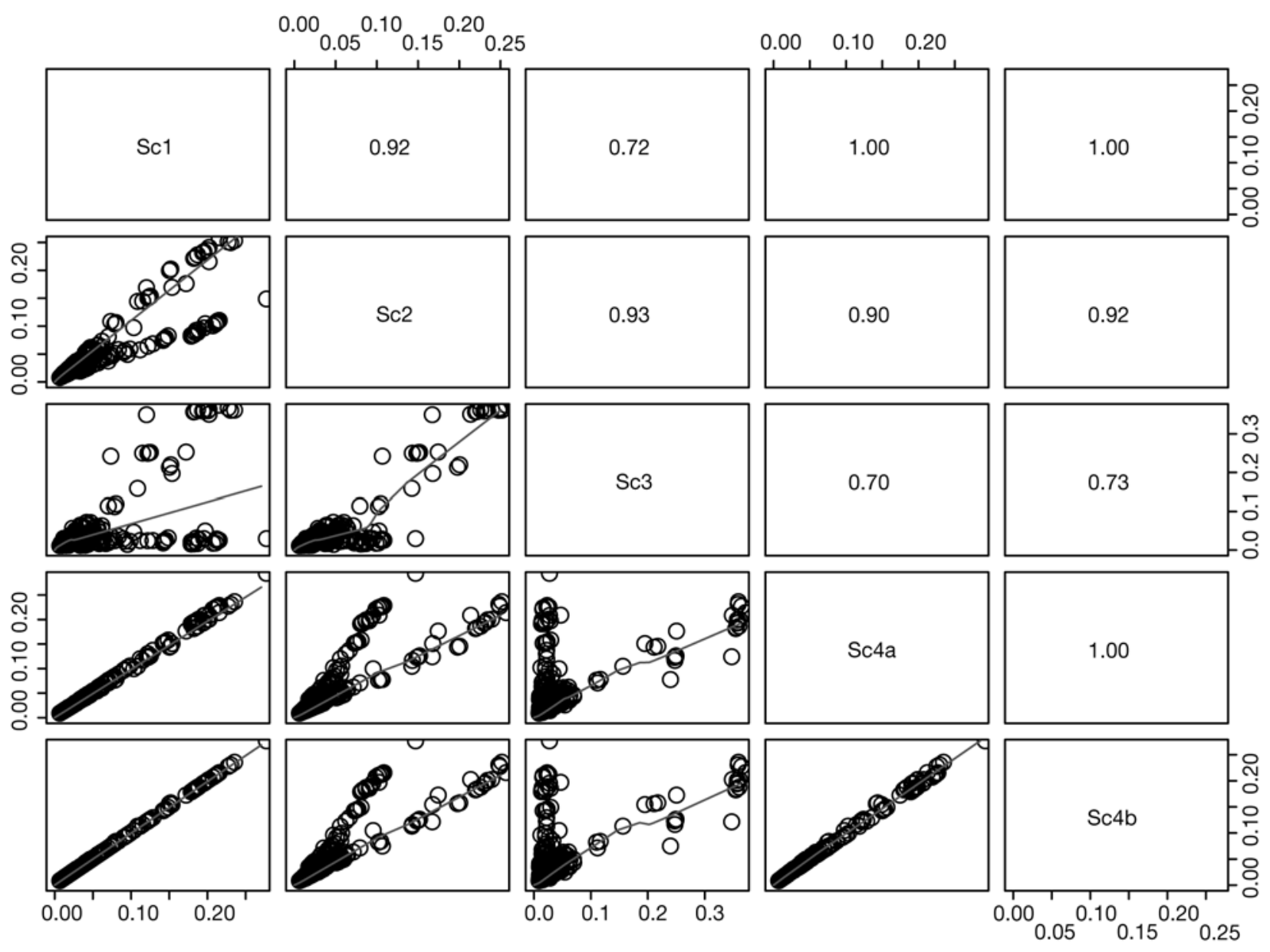

Fig. 5. Pairwise correlation between the different scenarios with calculated Pearson correlation coefficient. Sc $=$ Scenario

observed an overlap of the spatial extent of the areas of increased risk between the maps, but absolute risk values differed locally. This suggests that Scenario 2 resulted in relatively robust quantifications of risk. In the case of Scenario 3, we found clear differences between the 2 modified Scenario 3 results (Fig. 6). Where the OBS pressure was excluded, higher cumulative impact was predicted in areas to the south west. These areas of higher impact were not evident when the scenario was run with the EXT pressure excluded. This variation in Scenario 3 results suggested that Scenario 3 is more sensitive to the influence of single pressure categories.

To explore the effects of randomising the order of pressures in Scenarios 2 and 3, we fitted cubic smoothing splines to plots of raster values of the scenario with ranked pressures versus raster values from randomly ordered pressures (Fig. 7). The cubic smoothing splines reflect the relative agreement between the raster values of the scenario with ordered pressures and the raster values for the set of 6 randomised pressure rankings. If randomising of the respective sce- nario had resulted in identical predictions then all splines would follow a straight line. In other words, the greater the deviation from this straight line the greater the differences in spatial estimations of the modified scenario computations. We observed that for Scenario 2 (Fig. 7; left), there was a relatively low level of variation between the different scenario modifications. In contrast, we found a great level of variation between the raster values of Scenario 3 with ordered pressures and the 6 randomly ranked pressures. The results clearly suggested that Scenario 3 is more sensitive to the pre-defined pressure category $\times$ weight combinations than Scenario 2.

Using the concept of additive, synergetic and antagonistic effects, we compared the outcome for all cells of Scenario 1 to the other scenarios (Fig. 8). Scenarios 2 and 3 predicted higher values for cumulative impact than Scenario 1 for more than $60 \%$ of the study area. Although we found that Scenario 2 was less sensitive to changes in input parameters than Scenario 3, both outcomes suggest synergistic effects for the majority of the UK continental shelf. In contrast, Scenario 4a pro- 


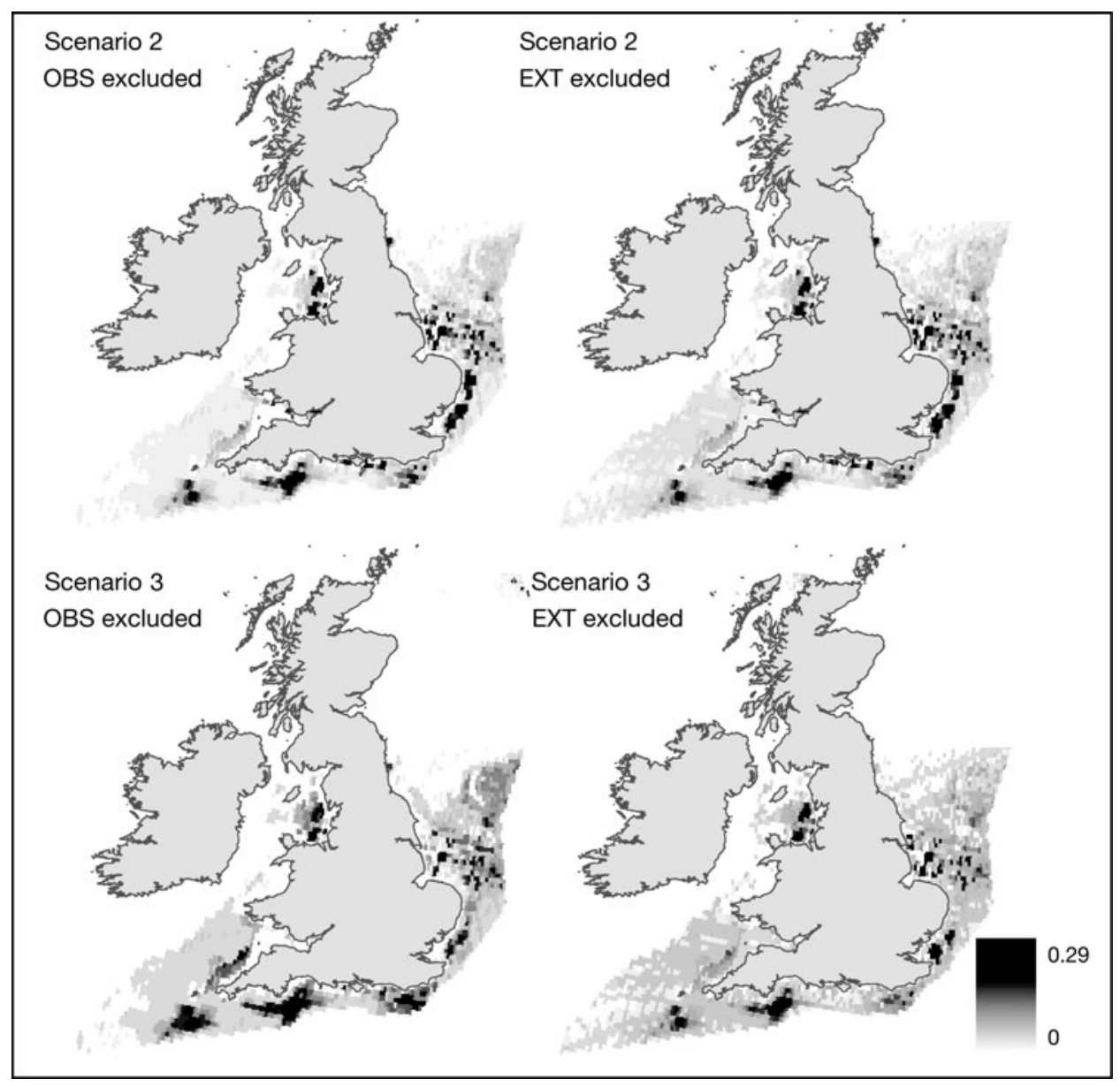

Fig. 6. Example results of the scenario evaluation. Top: Scenario 2 recalculated without the pressure category obstruction (OBS; left) and extraction (EXT; right). Bottom: Scenario 3 recalculated without the pressure category obstruction (OBS; left) and extraction (EXT; right). See Fig. 4 for scale details
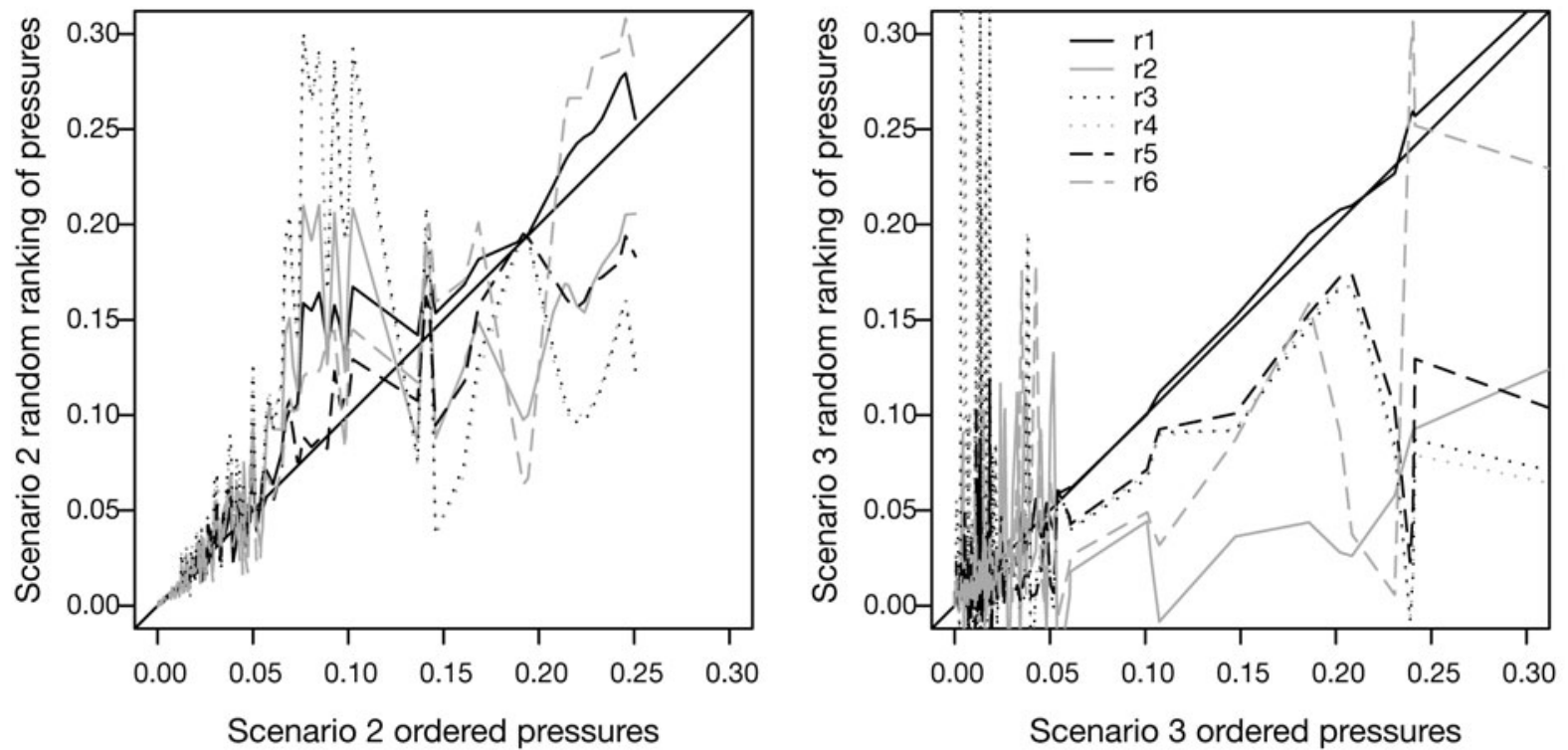

Fig. 7. Cubic smoothing splines fitted to the raster values of the ranked pressures of Scenarios 2 and 3 (see Table 3 ) and the raster values of the 6 random sets of pressure category $\times$ weight combinations (see Table 4) 


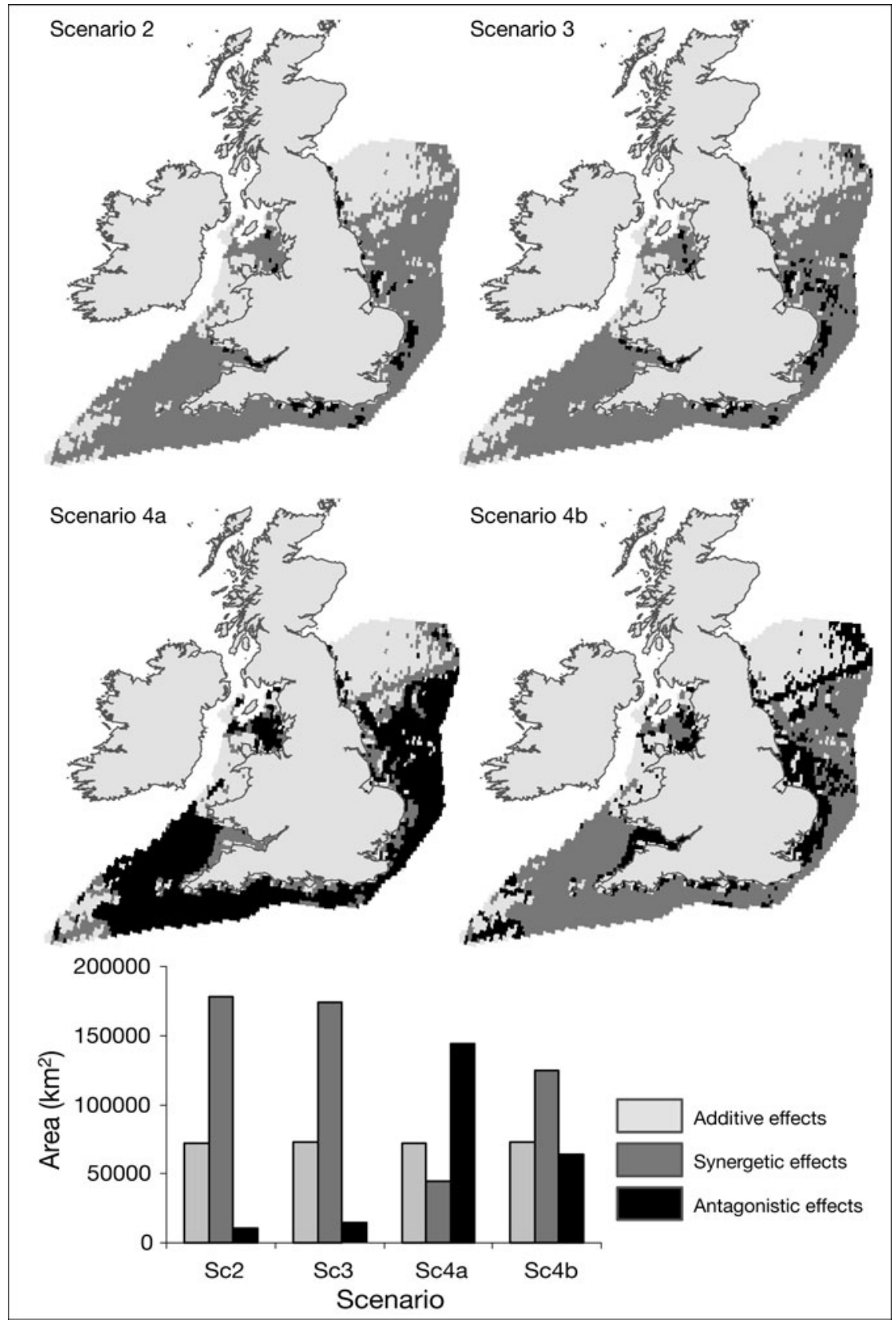

Fig. 8. Maps indicating the differences between reference Scenario 1 assuming additive effects as cumulative impacts and Scenario 2 (top left), Scenario 3 (top right), Scenario 4a (bottom left) and Scenario 4b (bottom right). Based on the definitions of synergy (cumulative impact value greater than sum of individual impacts) and antagonism (cumulative impact value lower than sum of individual impacts) (Folt et al. 1999), the differences to Scenario 1 outcomes were subjected to the respective categories (additive effects, synergistic effects, antagonistic effects)

duced lower values for cumulative impacts than those predicted by Scenario 1 for more than $50 \%$ of the area, thus suggesting antagonism. Interestingly, we found that values for cumulative impacts predicted by Scenario $4 \mathrm{~b}$ differed clearly from Scenario 1 for most of the study area, although scenario weights approximated to those of Scenario 1 (see Table 3).

\section{DISCUSSION}

This study aimed to develop a standardised framework to quantify the risk of cumulative impacts of human pressures in UK (England and Wales) waters. We focused on the critical evaluation of this framework and the assessment of variability and uncertainty in 
the modelling outputs rather than the development of precise marine planning scenarios.

The output of any modelling framework depends strongly on the quality of the input data. Hence, our list of human activities and pressures is not a comprehensive one, lacking data at a suitable scale on many activities affecting inshore areas, such as inshore fishing (see Eastwood et al. 2007, Stelzenmüller et al. 2008), recreational angling, navigational dredging or nutrient loading. As a consequence, our approach underestimated the relative risk of cumulative impact for inshore areas for each scenario. The contribution of watersheds to marine pressures is neglected in the presented application of our framework as we did not include data from e.g. land-based pollution. The inclusion of those land-based activities having an impact on the marine environment is essential for a robust estimate of the risk of cumulative impacts, especially in inshore waters. Nevertheless, our framework could be applied at any spatial scale and adapted to different pressure categories when suitable data are available.

A measure of the sensitivity of ecosystem components to pressures from human activities is essential (Hope 2006, Halpern et al. 2008a). We considered as an example the marine landscape categories as ecosystem components and converted respective measures of sensitivity from an ordinate scale to a numeric scale to derive sensitivity scores for each landscape category. Our algorithm computed impact on the landscape as a function of the intensity of a pressure and the measure of sensitivity. Consequently, an increased impact can be the result of either increased pressure intensity and a low level of sensitivity or a high level of sensitivity and a low pressure intensity.

Both measures introduced uncertainty into the modelling framework. We partly dealt with the uncertainty by using fuzzy logic to normalise the pressure layers, but some uncertainty remained due to the generalised measure of sensitivity. This uncertainty can only be quantified with the help of experimental studies with marine organisms to assess their sensitivity to the main pressures. This gap in experimental studies to quantify sensitivity or to ground-truth impact models was also recognised by Halpern et al. (2008b). Moreover, in the context of national marine planning, where decisions on resource allocations will be taken, it is crucial to develop a standardised measure of sensitivity of a suite of ecosystem components to generic human pressures (see e.g. Stelzenmüller et al. 2009).

An ecosystem approach to management using marine planning (Douvere 2008) requires a sound assessment of combined or cumulative impacts of several human pressures (Rosenberg \& McLeod 2005, Leslie \& McLeod 2007, Halpern et al. 2008a). This study aimed to contribute to the pressing questions on the quantification of cumulative impacts (Darling \& Côté 2008). In contrast to former studies (e.g. Ban \& Alder 2008), our framework comprised not only the mapping of pressures and additive impacts, but also quantified cumulative impacts according to the relative importance of single pressures. We constructed 4 different scenarios to quantify cumulative impacts and assessed the variability and sensitivity of model outputs. We based the reasoning of the different weighting schemes on the theory of different models for cumulative impacts (Folt et al. 1999). For instance, if pressures are applied consecutively to the marine landscapes rather than simultaneously, then the impact of the first pressure is likely to pre-condition the habitat to be less sensitive to the second pressure. Thus, the variability of the importance of individual impacts is reflected in our scenarios, allowing for a qualitative comparison of the model outputs. We applied the definitions of synergism and antagonism to compare Scenario 1 (assuming simple additive effects) with all other scenarios. This comparison is of importance when a potential user of this framework applies a scenario based on an alternative model. However, our framework did not model or describe the exact mechanisms of interactions leading to cumulative impacts. For instance a recent microcosm study indicated that pressures acting via similar mechanisms may result in additive effects, while those pressures that are dependent but act through different mechanisms result in synergistic effects (Mora et al. 2007). The development of models translating and extrapolating the mechanisms of synergistic or antagonistic interactions of pressures to large spatial scales remains for the future.

Our geospatial framework revealed a range of variability in the prediction of risk of cumulative impact that can be crucial at a local scale, such as in regional marine plans. For example, our scenario evaluation revealed that Scenario 3 with logistic criterion weights is more sensitive to the defined pressure $\times$ weight combinations than a linear weighting scheme and should therefore be used with caution unless the data clearly support the identification of one dominant pressure.

In general, we can conclude that the use of GISMCA, commonly deployed to assess decision alternatives (Malczewski 1999), is a useful approach to derive different quantifications of risk of cumulative impacts in the context of marine planning. It enabled us to develop a spatial explicit risk framework and to evaluate the robustness of the respective scenarios. The use of pairwise comparison to derive criterion weights enables expert opinion and/or stakeholder's perception to form input to the modelling of cumulative impacts, and provides a means of comparison and evaluation of diverse views. 
The UK recently stated its proposals to deliver a new marine planning system (Defra 2008). This will allow the development of marine plans enhancing protection of the seas while balancing pressures on the marine environment (Defra 2008). An effective marine planning system requires a number of steps, such as goal and objective setting, data collection, analysis and generation of spatial options, and development and evaluation of the marine spatial plan, followed by implementation of the plan (see Gilliland \& Laffoley 2008). Crucial to the integrated analysis phase is the quantification of cumulative impacts. Our framework can be used to conduct such an assessment, which leads to the question of how our framework could be put into effect for UK regional planning.

In principle, the framework can be applied for 2 types of planning scenarios: (1) there are no activities licensed within an area and a planner wants to assess the risk of cumulative impacts when licensing new activities, and (2) there are already activities licensed within the planning area and a planner needs to find a suitable site to licence a new activity. Based on the assumption that the first impact by a pressure causes the greatest mortality and with each subsequent impact only the remaining biota is affected, our scenario with equally weighted pressures can be used to assess the first planning option (1). Moreover, based on this assumption, the sensitivity score for each marine landscape would need to be adjusted for each additional pressure (planning scenario 2). Hence, as already discussed, such a relationship between sensitivity score and number of pressures/pressure types could only be established by experimental studies. As this information is lacking, a practical solution to find a suitable site to licence a new activity is the application of our framework Scenarios 2 or 3, using pressure category $\times$ weight combinations reflecting the importance of the pressures. One way of dealing with uncertainty with regard to pressure category $\times$ weight combinations would be the development of weighting schemes based on stakeholder consultations. At the same time, a planning cycle should allow for a rapid adaptation of spatial management decisions in case new knowledge on sensitivity and in-combination effects of human pressures becomes available.

Our framework is based on the assessment of impacts of generic pressures on ecosystem components developed and applied by OSPAR (Convention for the Protection of the Marine Environment of the North-East Atlantic) and also distinguished in the MSFD (EU 2008). Other studies dealing with the assessment of impacts of human activities (e.g. Ban \& Alder 2008, Halpern et al. 2008a) focus on the activities themselves rather than on generic pressures exerted by those activities. Thus, depending on the type of risk the user is interested in, our framework could also be modified to focus on multiple activities rather than on multiple pressures by omitting the first step. In this case, the sensitivity measure included in the framework would need to reflect the sensitivity to a certain activity as opposed to sensitivity to a pressure. Such a simplification of the framework might be suitable for local applications where comprehensive data on footprints and intensities of activities and the respective measures of sensitivities are available. In contrast, our more generic approach, using pressures rather than activities, allows a generic assessment and comparison between different spatial locations independent of the type of activities occurring in those areas.

In summary, uncertainty exists across many components of our modelling framework, including data quality, model design and/or parameters used. Nevertheless, management decisions must be made, even in the context of incomplete knowledge (Kerns \& Ager 2007). The computation of different scenarios of risk of cumulative impacts based on the users' assumptions also facilities a stakeholder engagement as different outcomes and their consequences can be discussed. Thus, different stakeholder views can be translated into respective risk assessment scenarios. We conclude that our spatially explicit, standardised and repeatable approach to quantifying risk of cumulative impact is a practical tool that can support the development of sustainable marine plans.

Acknowledgements. This study was funded by the UK Department of Environment, Food and Rural Affairs (Defra) (contracts ME1420 and A1148).

\section{LITERATURE CITED}

Ban N, Alder J (2008) How wild is the ocean? Assessing the intensity of anthropogenic marine activities in British Columbia, Canada. Aquat Conserv: Mar Freshw Ecosyst 18:55-85

Boroushaki S, Malczewski J (2008) Implementing an extension of the analytical hierarchy process using ordered weighted averaging operators with fuzzy quantifiers in ArcGIS. Comput Geosci 34:399-410

Burrough PA, McDonnell A (1998) Principles of geographical information systems. Oxford University Press, New York

- Christensen MR, Graham MD, Vinebrooke RD, Findlay DL, Paterson MJ, Turner MA (2006) Multiple anthropogenic stressors cause ecological surprises in boreal lakes. Glob Change Biol 12:2316-2322

Connor D, Gilliland P, Golding N, Robinson P, Todd D, Verling E (2006) UKSeaMap: the mapping of seabed and water column features of UK seas. Joint Nature Conservation Committee, Peterborough

Crain CM, Kroeker K, Halpern BS (2008) Interactive and cumulative effects of multiple human stressors in marine systems. Ecol Lett 11:1304-1315

> Darling ES, Côté IM (2008) Quantifying the evidence for ecological synergies. Ecol Lett 11:1278-1286 
Defra (2005) Charting progress: An integrated assessment of the state of the seas. Department for Environment, Food and Rural Affairs, London

Defra (2007) Cost impact of marine biodiversity policies on business - the marine bill. Department for Environment, Food and Rural Affairs, London

Defra (2008) Draft Marine Bill, April 2008. Defra, London

Douvere F (2008) The importance of marine spatial planning in advancing ecosystem-based sea use management. Mar Policy 32:762-771

Eastwood PD, Mills CM, Aldridge JN, Houghton CA, Rogers SI (2007) Human activities in UK offshore waters: an assessment of direct, physical pressure on the seabed. ICES J Mar Sci 64:453-463

EU (2008) Directive 2008/56/EC of the European Parliament and of the Council of 17 June 2008, establishing a framework for community action in the field of marine environmental policy (Marine Strategy Framework Directive). http://eur-lex.europa.eu/LexUriServ/LexUriServ.do?uri= OJ:L:2008:164:0019:0040:EN:PDF.

Evans KE, Klinger T (2008) Obstacles to bottom-up implementation of marine ecosystem management. Conserv Biol 22:1135-1143

Fisher P (2000) Fuzzy modelling. Taylor \& Francis, London

> Foden J, Rogers SI, Jones AP (2009) Recovery rates of UK seabed habitats after cessation of aggregate extraction. Mar Ecol Prog Ser 390:15-26

Folke C, Carpenter S, Walker B, Scheffer M, Elmqvist T, Gunderson L, Holling CS (2004) Regime shifts, resilience, and biodiversity in ecosystem management. Annu Rev Ecol Evol Syst 35:557-581

Folt CL, Chen CY, Moore MV, Burnaford J (1999) Synergism and antagonism among multiple stressors. Limnol Oceanogr 44:864-877

Gerwick BC, Gerwick BCJ (2007) Construction of marine and offshore structures. CRC Press, Boca Raton, FL

Gilliland PM, Laffoley D (2008) Key elements and steps in the process of developing ecosystem-based marine spatial planning. Mar Policy 32:787-796

Halpern BS, Selkoe KA, Micheli F, Kappel CV (2007) Evaluating and ranking the vulnerability of global marine ecosystems to anthropogenic threats. Conserv Biol 21:1301-1315

Halpern BS, McLeod KL, Rosenberg AA, Crowder LB (2008a) Managing for cumulative impacts in ecosystem-based management through ocean zoning. Ocean Coast Manage 51:203-211

Halpern BS, Walbridge S, Selkoe KA, Kappel CV and others (2008b) A global map of human impact on marine ecosystems. Science 319:948-952

Hastie TJ, Tibshirani RJ (1990) Generalized additive models. Chapman \& Hall, London

Hope BK (2006) An examination of ecological risk assessment and management practices. Environ Int 32:983-995

Jiang H, Eastman JR (2000) Application of fuzzy measures in multi-criteria evaluation in GIS. Int J Geogr Inf Sci 14: 173-184

Kerns BK, Ager A (2007) Risk assessment for biodiversity conservation planning in Pacific Northwest forests. For Ecol Manag 246:38-44

Kitsiou D, Coccossis H, Karydis M (2002) Multi-dimensional

Editorial responsibility: Jake Rice,

Ottawa, Ontario, Canada evaluation and ranking of coastal areas using GIS and multiple criteria choice methods. Sci Total Environ 284: $1-17$

Leslie HM, McLeod KL (2007) Confronting the challenges of implementing marine ecosystem-based management. Front Ecol Environ 5:540-548

$>$ MacDonald LH (2000) Evaluating and managing cumulative effects: process and constraints. Environ Manag 26: 299-315

Maina J, Venus V, McClanahan TR, Ateweberhan M (2008) Modelling susceptibility of coral reefs to environmental stress using remote sensing data and GIS models. Ecol Model 212:180-199

Malczewski J (1999) GIS and multicriteria decision analysis. Wiley \& Sons, New York

> Malczewski J (2004) GIS-based land-use suitability analysis: A critical overview. Prog Plann 62:3-65

> Malczewski J (2006) GIS-based multicriteria decision analysis: A survey of the literature. Int J Geogr Inf Sci 20:703-726

> Mora C, Metzger R, Rollo A, Myers RA (2007) Experimental simulations about the effects of overexploitation and habitat fragmentation on populations facing environmental warming. Proc Biol Sci 274:1023-1028

> Portman ME (2007) Zoning design for cross-border marine protected areas: The Red Sea Marine Peace Park case study. Ocean Coast Manage 50:499-522

> Rosenberg AA, McLeod KL (2005) Implementing ecosystembased approaches to management for the conservation of ecosystem services. Mar Ecol Prog Ser 300:270-274

> Ruckelshaus M, Klinger T, Knowlton N, DeMaster DP (2008) Marine ecosystem-based management in practice: Scientific and governance challenges. Bioscience 58:53-63

Saaty TL (1980) The analytical hierarchy process: planning, priority setting, resource allocation. McGraw-Hill, New York

> Stelzenmüller V, Rogers SI, Mills CM (2008) Spatio-temporal patterns of fishing pressure on UK marine landscapes, and their implications for spatial planning and management. ICES J Mar Sci 65:1081-1091

Stelzenmüller V, Ellis JR, Rogers SI (2009) Towards a spatially explicit risk assessment for marine management: Assessing the vulnerability of fish to aggregate extraction. Biol Conserv (in press), doi:10.1016/j.biocon.2009.10.7

Vaz S, Martin CS, Eastwood PD, Ernande B, Carpentier A, Meaden GJ, Coppin F (2008) Modelling species distributions using regression quantiles. J Appl Ecol 45:204-217

Villa F, Tunesi L, Agardy T (2002) Zoning marine protected areas through spatial multiple-criteria analysis: The case of the Asinara Island National Marine Reserve of Italy. Conserv Biol 16:515-526

Vinebrooke RD, Cottingham KL, Norberg J, Scheffer M, Dodson SI, Maberly SC, Sommer U (2004) Impacts of multiple stressors on biodiversity and ecosystem functioning: The role of species co-tolerance. Oikos 104:451-457

> Wood LJ, Dragicevic S (2007) GIS-based multicriteria evaluation and fuzzy sets to identify priority sites for marine protection. Biodivers Conserv 16:2539-2558

Zacharias MA, Gregr EJ (2005) Sensitivity and vulnerability in marine environments: An approach to identifying vulnerable marine areas. Conserv Biol 19:86-97

Submitted: June 18, 2009; Accepted: September 28, 2009

Proofs received from author(s): December 11, 2009 